\title{
A Case of Headache in the Context of a Spontaneous CSF Leak
}

ISSN: 2637-7748

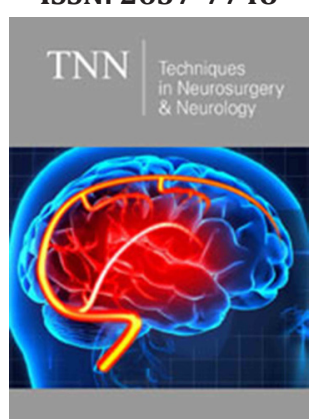

*Corresponding author: Nitin Butala, MD, MA, Baptist Neurology, 841 Prudential Drive, Jacksonville, FL 32207, USA

Submission: 海 November 22, 2019

Published:

Volume 3 - Issue 1

How to cite this article: Jessica C, Nitin B. A Case of Headache in the Context of a Spontaneous CSF Leak. Tech Neurosurg Neurol.3(1). TNN.000555.2019. DOI: 10.31031/TNN.2019.03.000555

Copyright@ Nitin Butala, This article is distributed under the terms of the Creative Commons Attribution 4.0 International License, which permits unrestricted use and redistribution provided that the original author and source are credited.

\author{
Jessica Cooper ${ }^{1}$ and Nitin Butala ${ }^{2 *}$ \\ Lake Erie College of Osteopathic Medicine, United States \\ Baptist Neurology, University, United States
}

\begin{abstract}
Headaches are one of the most frequent and nonspecific complaints of patients. A proper history and physical exam are crucial in properly diagnosing and treating a patient's headache. One rare cause of headaches is a cerebrospinal fluid (CSF) leak which is a phenomenon in which the cerebrospinal fluid surrounding the brain and spinal cord leaks out of the protective dural sac. This can be caused in response to trauma, but often occurs in the absence of any identifiable explanation. This is known as a spontaneous CSF leak and has an incidence of 5 per 100,000 per year making it easy to overlook in a patient presenting with headache. However, it is imperative to properly diagnose and treat patients with spontaneous CSF leak to ensure the best prognosis making it an important cause of headaches for medical providers to be aware of. Here we present a novel case of a 68-year-old African American female who was seen for a headache and was subsequently found to have a spontaneous CSF leak and was treated with an epidural blood patch. We also review the neuroimaging modalities used to localize and treat CSF leaks.
\end{abstract}

Keywords: CSF leak, headache, MRI, CT myelogram

Abbreviations: CSF: Cerebrospinal fluid; MRI: Magnetic Resonance Imaging; CT: Computed tomography

\section{Introduction}

According to the World Health Organization half to three quarters of adults aged 18-65 years in the world have experienced a headache in the last year. One rare cause of headaches seen in clinical practice are spontaneous CSF leaks. A spontaneous CSF leak is when the cerebral spinal fluid surrounding the brain and spinal cord leaks out of the protective dural sac with no identifiable cause. In contrast to CSF leaks due to trauma, a spontaneous CSF leak is idiopathic. It is often described in the context of orthostatic headaches and intracranial hypotension. Its incidence has been estimated as 5 per 100,000 per year, with a peak around age 40 years [1]. Diagnosis is made based on clinical suspicion and is confirmed using various imaging modalities including CT, MRI, and myelogram. Treatment involves placement of an epidural blood patch to seal the dural tears and repeat imaging. Here we present an interesting case of spontaneous CSF leak in a 68-year-old Female who presented to the neurologist complaining of headaches and a review of the imaging modalities used in the diagnosis of suspected leaks.

\section{Case presentation}

The patient is a 68-year-old right-handed African American female who was initially seen in the emergency department for headaches and nausea. She described the headaches as global and throbbing, and she noted that they were worse upon standing. Her workup included a CT which showed no abnormalities and an MRI of the brain that showed pachymeningeal enhancement, thought to be a nonspecific finding given her exam was stable and her inflammatory markers were negative. A repeat brain MRI one month later still showed pachymeningeal enhancement and also showed frontoparietal subdural hygromas not previously seen. The patient followed up at our neurology clinic where she still complained of headaches as well as added symptoms of tinnitus, upper extremity paresthesias and neck pain. A cervical spine x-ray was done and showed degenerative disc changes possibly responsible for her neck pain and paresthesias.

Due to the patients continued headaches as well as the persistent and worsening signal laboratory findings on brain MRI, we sent her back to the emergency department for a possible 
lumbar puncture and admission for further evaluation. However, she was sent back without lumbar puncture or admission. Her case was discussed with neurosurgery and neuroradiology conference and it was thought that this is more likely to be intracranial hypotension than an inflammatory or infectious etiology. Hence a recommendation to do MRI of the complete spine was given. The MRI of the spine showed evidence of CSF signal in the epidural space heightening our suspicion of a CSF leak. CT myelogram of the entire spine under fluoroscopic guidance was performed to localize the CSF leak. CT myelogram showed subdural fluid collections at multiple levels in the cervical and thoracic spine including C7 T1, T1 - T2 and T2 - T3, left nerve root sleeves. The presence of disc degeneration and bone spurs throughout these levels was thought to be a contributing factor. Epidural blood patches at C7$\mathrm{T} 1$ and T2-T3 were placed sublaminar under fluoroscopic guidance. The procedure was successful. On follow-up, the patient reported significant headache relief. She is scheduled for follow up imaging to confirm the success of the epidural blood patches.

\section{Discussion}

One rare cause of headache is spontaneous CSF leak. Often the patient will present with nonspecific findings such as headache and nausea as in our patient. Other findings in a patient with a CSF leak include neck pain or stiffness and tinnitus also seen in this patient as well as vertigo, dizziness, and many visual disturbances including but not limited to blurred vision, diplopia, and photophobia [2,3]. With this nonspecific presentation, the patients headaches may be confused for infectious or inflammatory causes including meningitis. Importantly, improper diagnosis of a spontaneous CSF leak can have life threatening sequelae for the patient. If a spontaneous CSF leak is left untreated there is a potential for brain herniation due to long term intracranial hypotension pulling the brain through the foramen magnum as well as from the CSF outside of the dural sac pushing down on the brain. Other potential complications of untreated CSF leaks include life-

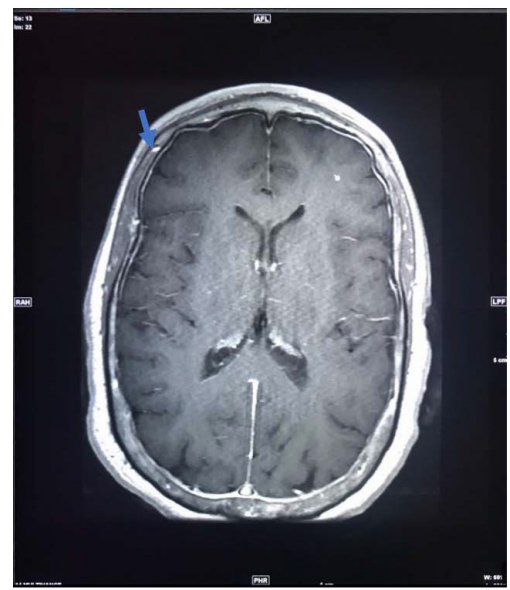

threatening meningitis, brain infection, or stroke. CSF leak should be suspected in patients who present with positional orthostatic headache that worsen upon sitting up from a lying position in the setting of spontaneous intracranial hypotension.

Four types of spontaneous CSF leak have been identified and categorized by the location and circumstances surrounding the leak. A type one CSF leak describes a leak caused by degenerative disc disease which creates a mechanical tear in the ventral dura [2]. This is the most common type of spontaneous CSF leak accounting for $48 \%$ of all leaks identified by one study [2]. Less common causes of spontaneous CSF leak include a more lateral tear in the dura arising from pre-existing thinned areas of dura (type 2), a CSF to venous fistula (type 3 ), or a distal nerve root sleeve that dissipates into the adjacent facial planes (type 4) [2].

Traditionally, MRI of the brain and spinal cord with and without contrast have been the imaging studies of choice when a spontaneous CSF leak is initially suspected. Prominent abnormal features including meningeal enhancement, sagging of the brain, and/or engorgement of cerebral venous sinuses on brain MRI (Figure 1,2 \& 3) or extra-arachnoid fluid collections, collapse of the Dural sac, or extradural extravasation of fluid on spinal MRI (Figure 4) may all be suggestive of a CSF leak [5-9]. If the leak can be adequately localized using MRI, an epidural blood patch is used to repair the leak. If the initial blood patch is unsuccessful a CT myelogram is performed to better localize the problem area and repair with an epidural blood patch is once again attempted. The difficulty of locating the site of a spontaneous CSF leak is that 30$40 \%$ of patients diagnosed will have leaks from multiple sites [4]. Furthermore, it has been noted that MRI imaging in patients with a suspected leak remains normal $20 \%$ of the time [1]. Spontaneous CSF leaks sometimes resolves spontaneously within weeks or years leaving patients with intermittent orthostatic headaches. Most, however, require multiple blood patches before culmination, and data suggests that $10 \%$ of patients will relapse regardless of treatment [1].

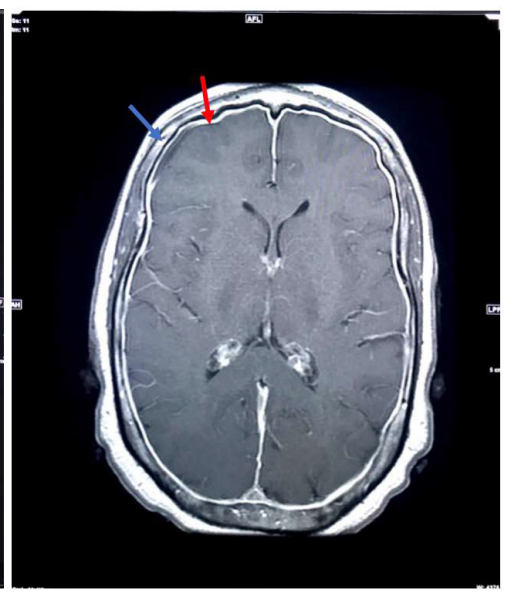

Figure 1: A comparison of an axial T2 weighted MRI brain of patient one month apart. a) Taken at first presentation of headache and shows dura tightly adhered to the calvarium and bridging veins tight against calvarium (blue arrow). b) Taken one month after initial presentation. Shows thickening of the meninges and pachymeningeal enhancement (red arrow) seen as a white ring surrounding the brain. Also shows bridging veins separating from calvarium (blue arrow). 

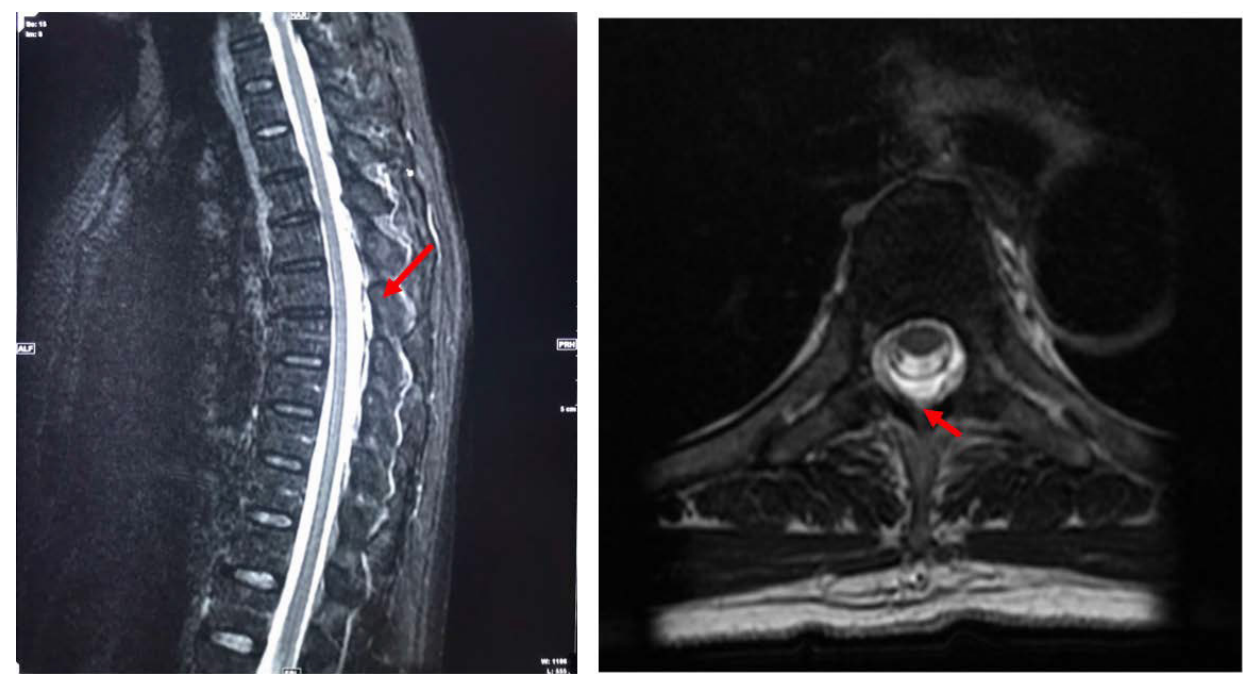

Figure 2: a) Saggital STIR MRI of the thoracic spine. STIR imaging saturates fat. High signal indicates fluid. In this case the high signal (red arrow) indicates epidural fluid outside of the dura mater confirming a CSF leak. b) axial T2 weighted MRI of thoracic spine demonstrating extradural CSF collection (red arrow).

\section{References}

1. Schievink WI (2006) Spontaneous spinal cerebrospinal fluid leaks and intracranial hypotension. JAMA 295(19): 2286-2296.

2. Farb R, Nicholson P, Peng P, Massicotte E, Lay C, et al. (2019) Spontaneous Intracranial Hypotension: A Systematic Imaging Approach for CSF Leak Localization and Management Based on MRI and Digital Subtraction Myelography. American Journal of Neuroradiology 40(4): 745-753.

3. Sun-Edelstein, Christina, Lay Christine (2019) Spontaneous intracranial hypotension: pathophysiology, clinical features, and diagnosis.

4. https://www.mdedge.com/neurology/migraineresourcecenter/ article/132780/headache-migraine /when-suspect-csf-leak

5. Mokri B, Piepgras DG, Miller GM (1997) Syndrome of orthostatic headaches and diffuse pachymeningeal gadolinium enhancement. Mayo Clinic Proceedings 72(5): 400-413.
6. Atkinson JLD, Weinshenker BG, Miller GM, Piepgras DG, Mokri B (1998) Acquired Chiari I malformation secondary to spontaneous spinal cerebrospinal fluid leakage and chronic intracranial hypotension syndrome in seven cases. Journal of Neurosurgery 88(2): 237-242.

7. Mokri B (2004) Low cerebrospinal fluid pressure syndromes. Neurol Clin 22(1): 55-74.

8. Starling A, Hernandez F, Hoxworth JM, et al. (2013) Sensitivity of MRI of the spine compared with CT myelography in orthostatic headache with CSF leak. Neurology 81(20): 1789.

9. https://utswmed.org/conditions-treatments/csf-leak/

For possible submissions Click below: 